\title{
TTR
}

Traduction, terminologie, re?daction

\section{(Ré)écriture du discours psychanalytique lacanien en traduction}

\section{Denise Merkle}

Volume 11, numéro 2, 2e semestre 1998

Psychanalyse et traduction : voies de traverse

Psychoanalysis and Translation: Passages Between and Beyond

URI : https://id.erudit.org/iderudit/037337ar

DOI : https://doi.org/10.7202/037337ar

Aller au sommaire du numéro

\section{Éditeur(s)}

Association canadienne de traductologie

ISSN

0835-8443 (imprimé)

1708-2188 (numérique)

Découvrir la revue

Citer cet article

Merkle, D. (1998). (Ré)écriture du discours psychanalytique lacanien en traduction. TTR, 11(2), 107-130. https://doi.org/10.7202/037337ar

\section{Résumé de l'article}

(Ré)écriture du discours psychanalytique lacanien en traduction - Cette étude se veut une réflexion sur l'apport de la réécriture des textes psychanalytiques de Jacques Lacan à la réception de sa pensée. Jean-René Ladmiral est d'avis que les textes psychanalytiques " difficiles ", de même que les textes philosophiques " profonds " et " obscurs ", se prêtent mal à une seule interprétation, invitant ainsi " plusieurs traductions ». Ces traductions différentes découlent inévitablement des lectures multiples résultant d'une riche intertextualité qui s'inspire de plusieurs disciplines : la médecine, la psychiatrie, la psychologie, la psychanalyse, la philosophie. Les influences germaniques (Freud, Husserl, Hegel) ainsi que françaises (Saussure, Lévi-Strauss, Sartre) contribuent également à tisser l'intertexte lacanien, rendu davantage complexe par les manifestations de l'inconscient dans le discours. $\mathrm{Au}$ lieu de condamner la multiplication de lectures et de traductions, nous proposons que ces lectures et traductions différentes enrichissent et approfondissent notre compréhension de la pensée complexe de Lacan.

Tous droits réservés ( $\odot$ TTR: traduction, terminologie, rédaction — Les auteurs, Ce document est protégé par la loi sur le droit d’auteur. L’utilisation des 1998 services d'Érudit (y compris la reproduction) est assujettie à sa politique d'utilisation que vous pouvez consulter en ligne.

https://apropos.erudit.org/fr/usagers/politique-dutilisation/ 


\section{(Ré)écriture du discours psychanalytique lacanien en traduction}

\section{Denise Merkle}

[....] il est difficile de nier que toutes les traductions ne sont pas excellentes [...] et particulièrement en ce qui concerne les textes difficiles de la psychanalyse, de la philosophie ou des sciences humaines en général. Un grand philosophe, obscur et profond, comme Hegel ou Heidegger, par exemple, pose à son traducteur des problèmes d'interprétation presque insolubles, et l'on comprend dès lors qu'il soit proposé plusieurs traductions différentes et que ces dernières deviennent un enjeu de controverses dans la discussion philosophique, comme autant de versions différentes d'un même texte original ressortissant chacune à une interprétation spécifique.' (Ladmiral, 1979, p. 92)

\section{Réception de Lacan}

En France, les écrits du " Freud français "Jacques Lacan ont inspiré des polémiques à cause des lectures multiples que son cuvre a suscitées. D'une part, il y a une lecture philosophique qui découle du dialogue que Lacan a engagé avec certains philosophes dans ses propos. Alain

I Je souligne. 
Juranville et Paul-Laurent Assoun ${ }^{2}$, indépendamment l'un de l'autre, font des rapprochements entre la pensée philosopho-psychanalytique de Lacan sur la relation entre le sujet et l'autre/l'Autre, et la pensée d'Emmanuel Levinas sur la responsabilité, voire le devoir éthique de l'individu envers l'Autre. Le rapprochement de la philosophie et de la psychologie (voire de la psychanalyse) se conforme ainsi à une tradition qui regroupe ces deux disciplines, bien que la psychanalyse en tant que " science de l'inconscient " confirme la naissance des " sciences de l'homme ", tout comme Assoun la conçoit dans Freud et les sciences sociales : Psychanalyse et théorie de la culture (1993) ${ }^{3}$. D'autre part, il y a une lecture féministe qui représente une réaction contre certains propos de

${ }^{2}$ La renommée de Paul-Laurent Assoun s'est établie grâce à Freud, la philosophie et les philosophes dont la première édition date de 1976, et celle d'Alain Juranville gráce à la publication de Lacan et la philosophie (1984).

${ }^{3}$ Selon le Oxford Companion to Philosophy (1995, pp. 727-728) le divorce officiel entre la philosophie et la psychologie date du milieu du vingtième siècle quand les associations professionnelles et les départements d'université devinrent distincts. En ce qui a trait à la psychanalyse, les philosophes débattent depuis longtemps l'appartenance disciplinaire de la psychanalyse : est-ce une science, une pseudo-science ou sui generis? Les analystes, dont le "père "de la psychanalyse, semblent ne pas être convaincus de l'emplacement intellectuel de leur discipline. À partir de 1895, "Freud attempted to draw up an unadulterated picture of psychoanalysis as a natural science; but shortly after, he abandoned the Project and never resumed it in an uninterrupted way" (Mahony, 1994, p. 319). Benvenuto et Kennedy (1985, pp. 20-21) affirment que " Lacan seems to be quite clear that Freud was moving into the area of what are now called the "human sciences", thase disciplines essentially concerned with human meaning ". D'ailleurs dans Les quatre concepts fondamentaux de la psychanalyse, Lacan pose les questions * La psychanalyse est-elle une science? " et "Qu'est-ce qu'une science qui inclut la psychanalyse? ", répondant que la psychanalyse est la " science de l'inconscient " comme la linguistique est la science du langage, l'incluant ainsi dans les sciences humaines. Ces deux questions figurent dans le Résumé rédigé pour l'Annuaire de l'École pratique des Hautes Études 1965, Le Séminaire Livre $X I$ (en-dos). Lacan pose la première question dans Le Séminaire (p. 47). A la page 185, il dit : * Si la psychanalyse doit se constituer comme science de l'inconscient, il convient de partir de ce que l'inconscient est structuré comme un langage. * 
Lacan. Luce Irigaray et Julia Kristeva élaborent des théories féministes à partir de leurs lectures du " maître ", tout en remettant en cause des hypothèses fondamentales, dont le patriarcat, le symbolique et l'imaginaire, qui sous-tendent la théorisation lacanienne. Déjà dans la société d'où émane le texte, chaque lecteur ou chaque lectrice se fie à son interprétation personnelle du texte de départ, créant ainsi " les versions différentes d'un mêtme texte original "(Ladmiral, 1979, p. 92).

La vague de l'intérêt pour ce " retour à Freud " dans un cadre structuraliste ${ }^{4}$ - à l'encontre du « retour à Freud $n$ dans un cadre marxiste à la Althusser, à la Fromm ou à la Marcuse - , a traversé l'Atlantique dans les années soixante, puisque le discours de la psychanalyse offrait un nouveau système de réflexion. Ceci et l'unilinguisme de beaucoup d'Américains ont contribué à la production de traductions en langue américaine de certains écrits de Lacan. Mais la traduction ne se limitait pas à ses écrits. Au contraire, on a peu après entamé également la traduction de la pensée critique inspirée par ses écrits en France, comme par exemple, les œuvres d'Irigaray, Kristeva', Assoun et Juranville, entre autres.

Toujours est-il qu'en Amérique on a tendance à faire une lecture littéraire ou culturelle à partir des traductions de l'ouvre de Jacques Lacan. Comme le constate Testa (1997, p. D9), c'est surtout grâce à " cette adaptation " que la pensée de Lacan est diffusée. Des publications encore récentes dans le domaine littéraire confirment en effet la diffusion de sa pensée: Patriarchal Desire and Victorian Discourse : A Lacanian Reading of Anthony Trollope's Palliser Novels de Priscilla L. Walton (Toronto, University of Toronto Press, 1995) et Margaret Atwood's Power: Mirrors, Reflections and Images in Select Fiction and Poetry de Shannon Hengen (Toronto, Second Story Press, 1993). Ces " lectures littéraires "sont quand même, selon certains, superficielles par moments, puisqu'elles se contentent trop souvent de ne retenir que

"Todd Dufresne (dir.), Returns of the "French Freud" : Freud, Lacan and Beyond New York/Londres, Routledge, 1997.

s Voir Sherry Simon, Gender in Translation (1996, pp. 86-110), pour une discussion sur quelques erreurs trouvées dans les traductions américaines de Cixous, Kristeva et Irigaray. 
certaines notions du discours lacanien, telles les " stade du miroir, nœud borroméen, discours du maître, le symbolique, l'imaginaire ", les isolant ainsi de leur contexte théorique psychanalytique. Il est également admis que les milieux psychanalytiques américains n'acceptent la psychanalyse lacanienne qu'avec réticence, privilégiant depuis des décennies l' " ego psychology ". Bref, malgré l'intérêt apparent, la pensée lacanienne connaît une dissémination beaucoup plus restreinte et une analyse parfois fragmentaire de ce côté de l'Atlantique, l'intérêt s'avérant essentiellement littéraire ou philosophique.

Benvenuto et Kennedy (1986) discutent de cette contextualisation des écrits de Lacan dans leur introduction à The Works of Jacques Lacan. Benvenuto, qui a un doctorat en philosophie, traite de l'effet de la différence culturelle sur la réception parfois hostile qui attend Lacan en Grande-Bretagne et en Amérique si on la compare à sa réception en France. Benvenuto et Kennedy croient que les lecteurs de Lacan en Angleterre et en Amérique, surtout avant les années quatre-vingt-dix, le considèrent avec mépris à cause de son discours qui les déconcerte, ce qui les fâche ou les irrite. Bien que les lecteurs français trouvent aussi son discours psychanalytique provocateur, leur réaction n'est pas celle du mépris. La réception mitigée des Français comparativement à la réception plutôt négative des Anglo-Saxons reflète une différence culturelle fondamentale entre l'Angleterre et l'Amérique d'un côté, et le Continent de l'autre, celui-ci ayant une orientation davantage toumée vers l'interdisciplinarité. Compte tenu de cette aversion anglo-saxonne jusqu'aux années quatre-vingt-dix envers le discours interdisciplinaire - à l'heure actuelle on constate un revirement dans les milieux universitaires américains et canadiens - et non transparent ${ }^{6}$, il fallait présenter la pensée de Lacan sous une lumière favorable afin de briser la résistance naturelle anglo-saxonne contre son style, pendant que l'on tentait de rendre fidèlement le contenu. Il fallait également réussir à donner au lecteur le goût pour l'arrière-plan gallique et, jusqu'à un certain

' Benvenuto et Kennedy affirment que sur le Continent on considère les philosophes de langue allemande Hegel, Heidegger et Husserl, entre autres, d'une importance fondamentale, et on leur consacre le temps nécessaire afin de pénétrer leur pensée. Par contre, on les considère inutilement obscurs en Angleterre et en Amérique (Benvenuto et Kennedy, 1986, p. 13). 
point, germanique de la pensée de Lacan (Benvenuto et Kennedy, 1986, p. 13).

Si, pour le lecteur américain, les écrits de Lacan étaient d'un intérêt surtout littéraire ou philosophique - sans tenir compte de l'analyse psychanalytique de textes que sa pensée permet -, l'approche traductionnelle devrait-elle être celle d'un texte " difficile ", et plusieurs traductions d'un seul texte donc permises, nécessaires même? Il ne faut pas oublier que déjà en français ses écrits permettent de multiples lectures... De prime abord, il semblerait alors que plusieurs traductions soient de mise. Nous savons qu'à l'encontre de la traduction littéraire qui "n'est jamais qu'une "version" du texte original, une parmi d'autres ", la retraduction d'un texte psychanalytique, c'est-à-dire " scientifique ", implique que la traduction que l'on remplace soit jugée défectueuse, ceci à moins que le texte de départ ne soit jugé " difficile ", c'est-à-dire " obscur et profond ", pour reprendre les mots de Jean-René Ladmiral (1979, p. 92). La France, la Grande-Bretagne et l'Amérique croient effectivernent que les textes de Lacan sont difficiles à décoder. Robert Larose en citant Ladmiral affirme que les textes difficiles à décoder sont difficilement " objectivables " (cf. Ladmiral dans Larose 1987, pp. 240242). Toujours est-il que les caractéristiques socio-culturelies - c'est-àdire la tolérance envers certains types de discours - de la société d'où émane le texte sont en l'occurrence très différentes comparativement à celles de la culture d'arrivée. Et les stratégies de traduction sélectionnées pour compenser le niveau de tolérance inférieur de la culture d'arrivée diffèrent d'un traducteur à l'autre.

Or bien qu'il existe plus d'une traduction de Fonction et champ de la parole et du langage en psychanalyse de Lacan - mentionnons celles d'Anthony Wilden et d'Alan Sheridan, entre autres -, la version à laquelle on a tendance à se référer dans le cadre des études supérieures en lettres anglaises est celle de Sheridan. Mais est-elle en réalité une meilleure traduction, remplaçant celle qui la précédait, défectueuse ? Ou bien, si nous admettons que le texte de départ est d'une grande difficulté, la multiplication des traductions contribuera-t-elle dans ce cas-ci à en dégager le sens profond dans une perspective benjaminienne? Dans ce cas y aurait-il lieu de parler en termes de meilleure traduction, ou bien de 
" traduction canonique ${ }^{7}$ " qui fasse autorité? Si l'on se contente tout simplement de lire un texte de Lacan, la question a en réalité peu d'importance. Pourtant, elle devient pertinente lorsqu'on traduit un ouvrage dont le sujet est la pensée de Lacan dans le cas où le traducteur voudrait se servir d'un discours lacanien reconnaissable (voire normalisé) en langue américaine'. $\dot{A}$ titre d'exemple, le lecteur d'Assoun ou de Juranville ne souhaite pas nécessairement être entraîné dans une * discussion philosophique " découlant des " traductions différentes " (Ladmiral, 1979, p. 92) des écrits de Lacan ou de Freud qui ont été proposées, comme par exemple le choix entre Other, other(s), other(s) pour rendre " Autre (grand Autre), autre(s) (petit autre), autrui ", le choix entre ego/self pour rendre " Moi/moi ", le choix entre instance, insistence ou agency pour rendre a instance ", le choix entre l'emprunt d'un terme français lorsque le terme s'avère a intraduisible "d'après certains, ou l'insistance sur la possibilité de trouver un équivalent dans la langue d'arrivée (pensons à la difficulté de traduction que les termes " méconnaissance n et " jouissance ${ }^{q}$ " présentent). En revanche, cette discussion intéresse certains traducteurs/chercheurs, dont Ladmiral qui

\footnotetext{
${ }^{7}$ Marilyn Gaddis Rose a introduit cette notion lors de la discussion qui a suivi la première version de cette communication donnée à Saint-Jean, Terre-Neuve, le 3 juin 1997.
}

"À l'heure actuelle la bibliothèque en langue anglaise consacrée à l'étude de la pensée de Lacan prend de l'ampleur. Aux titres de la bibliographie, grâce à Testa (1997, p. D9) on peut ajouter : Althusser, « Freud et Lacan w, dans Lenin and Other Essays (New Left Books, 1971); Dylan Evans, An Introductory Dictionary of Lacanian Psychoanalysis (Routledge, 1996); Elizabeth Grosz, Jacques Lacan - a Feminist Introduction (Routledge, 1996); Elisabeth Roudinesco, Jacques Lacan \& Co: A History of Psychoanalysis in France, 1925-1985 (University of Chicago, 1990) et Jacques Lacan, tr. Barbara Bray (Columbja University, 1997). Cette liste n'est pas exhaustive.

"Madan Sarup, Jacques Lacan (1992, pp. 129-130) : « Jouissance is a notoriously polysemic term in Lacan. It denotes both the possession-enjoyment of an object capable of giving pleasure. In popular registers it means "orgasm", with jouir as the equivalent of "to come". But jouir can antiphrastically (use of a word to imply the exact opposite of its normal meaning) come to refer to the experience of exquisite pain which occasions a momentary loss of consciousness. It can also comprise an element of horror, a highly eroticised death drive that goes far beyond the pleasure principle. * 
décrit, par exemple, une difficulté terminologique dans la traduction du discours psychanalytique lorsqu'il fait le rapprochement entre les difficultés de traduction de textes psychanalytiques et de textes philosophiques dans son ouvrage Traduire : Théorèmes pour la traduction :

Il suffit de penser à la révolution terminologique qui a traversé (bouleversé) les milieux psychanalytiques parisiens, il n'y a pas si longtemps, et au terme de laquelle il ne peut plus être question de traduire le Trieb freudien autrement que par pulsion - alors que des décennies d'ignorantisme s'étaient contentées de parler d'instinct. (Ladmiral, 1979. pp. 91-92)

Il convient maintenant d'explorer pourquoi certains qualifient les écrits de Lacan de textes " difficiles ", en regardant de plus près l'intertexte du discours de Lacan et la nature du discours psychanalytique afin de mieux apprécier la complexité, la subtilité et la qualité littéraire de ce discours.

\section{Intertexte}

Après avoir soutenu avec succès en 1932 une thèse de doctorat en médecine (Charbonneau, 1997, p. 74), Lacan a entamé des recherches en psychologie, qu'il a plus tard abandonnées. Pendant cette même période, il a lu Husserl et, s'inspirant de la phénoménologie, a élaboré une théorie de la "psychologie intentionnelle ", où il a mis l'accent sur la compréhension de l'histoire du sujet/patient (Charbonneau, 1997, pp. 7576). De plus, au début des années trente, il a commencé à s' intéresser aux activités artistiques et intellectuelles des surréalistes. La réaction positive de Salvador Dali à sa thèse de doctorat sur la paranoïa a renforcé les affinités marquées de Lacan avec le surréalisme, lui aussi préoccupé par le travail de l'inconscient. À partir de 1933, il a publié des articles dans Le Minotaure, revue surréaliste. Il est devenu membre de la Société psychanalytique de Paris en 1934, et a fait paraître en 1932 une traduction de Freud (1922) qu'il a signée (" De quelques mécanismes névrotiques dans la jalousie, la paranolia et l'homosexualité ») (Charbonneau, 1997, p. 288). Il semble que Freud ait laissé les traces les plus profondes, comme il avait également influencé les surréalistes. Néanmoins, puisque depuis le début du siècle la réception française de la psychanalyse viennoise était peu chaleureuse, Lacan a dû " rendre la doctrine plus 
conforme au " génie latin" 10 (Charbonneau, 1997, p. 71).

Marie-Andrée Charbonneau, elle-même titulaire d'un doctorat en philosophie, a étudié l'intérêt de Lacan pour la pensée philosophique dans son ouvrage Science et métaphore: Enquète philosophique sur la pensée du premier Lacan (1926-1953). Selon elle, Lacan, qui se considérait toujours médecin de la psyché humaine, a traversé différentes phases, d'abord psychiatre (1926-1932), puis psychologue (1933-1939), psychanalyste (1945-1953), et, enfin, philosophe. Charbonneau a montré que la pensée non seulement de Freud, mais aussi celles de Husserl, de Sartre, et de Lévi-Strauss avaient marqué son évolution. Bref, selon Charbonneau, Lacan s'est inspiré - parfois très directement et librement - des écrits de Freud (libido, inconscient, Imago), de Husserl (phénoménologie), de Wallon (complexe d'intrusion, stade du miroir), de Hegel (maître-esclave), de Sartre (expérience vécue, l'imaginaire devient la vraie réalité), de Lévi-Strauss (loi d'Eedipe, consensus scientifique, fonction symbolique) et de Saussure (structure du langage).

La compréhension, même rudimentaire, de la pensée lacanienne suppose donc une familiarité poussée avec ce vaste intertexte. Déjà en 1973, Wilden était d'avis qu'afin de comprendre les écrits de Lacan, le lecteur devait avoir

a more than usual intimacy with the texts of Freud [...], an acquaintance with Hegel and his French commentators [...], a familiarity with the early Heidegger and the early Sartre [...]. a knowledge of the concepts of modern structural linguistics (Saussure, Jakobson) and structural anthropology (Mauss, Lévi-Strauss). (Wilden, 1973, p. viii).

Madan Sarup décrit cet intertexte autrement :

${ }^{10}$ Marie-Andrée Charbonneau explique que pour Pichon "la francisation des concepts freudiens revêt une importance primordiale. Elle cite Laforgue et Pichon ( De quelques obstacles à la diffusion de la psychanalyse *, 1923, p. 293) : « Par exemple, il est impossible à un Français de ne pas associer libido avec libidineux. Libido est de ce fait même un mot qui doit être banni de la nomenclature psychanalytique française, pour être remplacé par un vocable mieux approprié à rendre l'idée beaucoup plus générale que les freudistes veulent lui faire exprimer $*$ (Charbonneau, 1997, p. 72). 
Fully to understand Lacon's thought and his enormous cultural influence we must have a look at a wide range of discourses: Saussurean semiology; Jakobsonian rhetorical analysis; Freud's CEdipal schema; Benveniste's linguistics; Lévi-Strauss's idea of women as exchange; Hegel's concept of desire... (Sarup, 1992, pp. ix-x).

Toujours est-il que le tissage de cet intertexte s'avère complexe. Par ailleurs, il y a permutation dans cette intertextualité - comme, par exemple, lorsque Lacan donne * une interprétation tout à fait personnelle ", "qui aboutit à transformer la pensée de Freud " (Kassaï et Ladmiral, 1988, p. 42) - qui ne fait que multiplier les obstacles à la traduction. De toute évidence, la lecture de Lacan (et, par extension, la traduction de ses écrits) suppose une familiarité avec ce vaste champ dans lequel se meut la pensée théorique de Lacan en évolution continue. Cependant, selon John Muller et William Richardson dans Lacan and Language: A Reader's Guide to Écrits, même une certaine familiarité avec cet intertexte $n$ 'en garantit pas pour autant la compréhension, bien qu'elle foumisse suffisamment d'indices pour rendre certaines lectures ou interprétations (voire solutions de traduction) plus probables que d'autres.

\section{Nature du discours psychanalytique}

Au premier abord le style figuratif et les allusions souvent littéraires de Lacan pourraient laisser entendre qu'il était un littéraire. Par ailleurs, certains considèrent déjả les écrits de Freud comme de la littérature ou, au moins, on apprécie - tout comme Ladmiral apprécie * ce grand styliste de l'allemand qu'était Freud " (Kassai et Ladmiral, 1988, p. 4) - les qualités stylistiques et littéraires de ses écrits. Freud lui-même était d'avis que le discours de la psychanalyse était un langage déciđément figuratif (eine Bildersprache). Il est important de noter que pour Freud :

[...] the language of psychoanalytic theory was out and out a figurative language (Bildersprache) and not a literal one, and more than that, only by means of that distorting figurative language could [he] know about unconscioss processes, not to mention the fact that their distorting nature can never be fully known in the first place. (Mahony, 1994, p. 317)

Tout comme Freud avant lui et les surréalistes, Lacan était aussi d'avis que le langage psychanalytique était figuratif et lié au travail de 
l'inconscient. Ses textes risquent ainsi de laisser croire qu'il voulait en effet que son style soit obscur et ses énoncés cryptiques tout comme l'inconscient. Selon Benvenuto et Kennedy,

Lacan evolved a style of writing whose aim was to avoid being oversystematized and reductive, and to reflect the workings of the unconscious. Lacan's prose thus often obeys the laws of the unconscious as they were formalized by Freud - it is full of puns, jokes, metaphors, irony and contradictions, and there are many similarities in its form to that of psychotic writing. (Benvenuto et Kennedy, 1986, p. 12)

Benvenuto et Kennedy ajoutent : " [t] his makes reading his essays an intellectual task of some magnitude, on a par with reading Finnegan's Wake [sic] " (p. 12), et certains-du monde francophone comme du monde anglophone - qualifient ses écrits d'indéchiffrables.

Parler d'écriture psychotique est sans doute exagéré; toutefois, le discours lacanien contient bien des énoncés peu clairs, voire ambigus, qui risquent de porter à confusion. Une allusion à l'ambigulté d'un mot français dans le Séminaire $I X$ de 1964 : Les quatre concepts fondamentaux de la psychanalyse se trouve traduit en anglais par "the superb ambiguity of the French language" " (1973, p. 55). Cette lecture porte le lecteur à croire que la langue française (voire la psyché française) est déjà particulièrement apte à rendre le discours psychanalytique de Lacan, tout comme certains pensaient (et peut-être pensent encore) que la langue allemande (voire la psyché germanique) était particulièrement apte à rendre le discours psychanalytique de Freud. Cependant, cette ambiguitté qui caractérise le discours psychanalytique coûte parfois cher.

John Muller et William Richardson explicitent le prix de cette ambiguitté : en effet, tout lecteur, qu'il soit traducteur ou non, risque de ne

"Ce qui n'est pas exactement ce que Lacan a dit, comme la citation sujvante le confirme : * $\dot{A}$ cette exigence répondent ces points radicaux dans le réel que j'appelle rencontres, et qui nous font concevoir la réalité comme unterlegt, untertragen. ce qui en français se traduirait par le mot même, en sa superbe ambiguîté dans la langue française, de souffrance. La réalité est là en souffrance, là qui attend "(Lacan, 1973, p. 55). 
pas comprendre le texte original puisque les Écrits lui offrent, comme les rêves de Freud, un puzzle. Selon eux, essayer de comprendre ce puzzle, c'est jouer aux devinettes (Muller et Richardson, 1982, p. 415). Par conséquent, le traducteur ne peut pas être convaincu que sa lecture soit la bonne; il se rend ainsi déjà compte qu'il se peut que la solution proposée soit une parmi d'autres. Wilden, traducteur, est même porté à se demander si, dans sa traduction de Lacan, il explique ce qui est déjà évident ou s'il ajoute à l'ambiguìté de ce qui est déjà ambigu (Wilden, 1973, p. vii). Ailleurs, il accuse Lacan d'avoir un style précieux, quelque chose que le lecteur français accepte comme faisant partie de sa tradition littéraire, mais que des lecteurs anglophones trouvent étranger et parfois offensant. Wilden s'explique :

Lacan's constitutional predisposition to ambiguity, sometimes even on insignificant points, makes him difficult enough in French. where a tradition of préciosite gives him far greater latitude than is the case for the writer in English. And over the years since 1953, he seems progressively to have become a prisoner of his own (dense and allusive (vii)] style. (Wilden, 1973, p. ix)

Par ailleurs, Benvenuto et Kennedy accusent Lacan d'avoir refusé de définir tous ses termes - mais il se peut que cet état des choses soit * une conséquence des conditions du discours psychanalytique ${ }^{12}$ " rendant ainsi les notions très floues et malléables -, et d'avoir modifié le contenu sémantique de quelques termes freudiens ${ }^{13}$. Par la suite, le sens

12 Jean-René Ladmiral ajoute les précisions suivantes : " Le propre de l'expérience analytique, et du langage auquel elle donne lieu, est de s'instaurer par référence exclusive, et en quelque sorte "circulaire", à un univers de discours qui l'institue autant qu'elle la fonde. [...] Il s'agit en effet d'un métalangage - où, en termes linguistiques, il n'y a d'autre référent que le signifié lui-même * (Ladmiral, 1979, p. 252).

${ }^{13}$ Susanne Hommel dans son article intitulé * Wo Es war, soll Ich werden : interprétations lacaniennes " montre que la * traduction " proposée par Lacan, ainsi que ses commentaires sur la traduction, change plus de sept fois : " Là où le $\mathrm{S}$ était, là le Ich doit être " (1954-1955) ; * Là où c'était, là Je doit advenir " (19581959); * Là où c'était - Je dois devenir * (1967-1968), entre autres (Kassaï et Ladmiral, 1988, pp. 6-9). Plus loin, Ladmiral écrit : * Lacan a donné de certains concepts freudiens une interprétation tout à fait personnelle, qui aboutit en fin de compte à transformer la pensée de Freud * (Kassaì et Ladmiral, 1988, p. 42). 
et la fonction de ses termes ont évolué au cours des années avec peu d'explication (Benvenuto et Kennedy, 1986, pp. 12-13). Par conséquent, son insouciance apparente face à la rigueur terminologique ${ }^{14}$ crée des textes " à faible redondance ${ }^{15}$ ". Puisque le nombre de signes est relativement petit et que le signifié de ces termes (par exemple, moi, autre, symbolique) change, la charge sémantique devient très lourde, et les " signes communiquent une grande quantité d'information " (Larose, 1987 , p. 247). De plus, la tradition freudienne dans le discours psychanalytique français a été soulignée par Ladmiral, le discours psychanalytique viennois témoignant d'une terminologie connotative tirée de plusieurs registres et disciplines ${ }^{16}$. Si dans les écrits de Lacan il y a en fait très peu de redondance puisque le sens des mots change, l'éventail de l'interprétation deviendra alors plus grand. Une pléthore de lectures, d'interprétations et de traductions s'avère donc inévitable. Tout compte fait, toutes ces traductions possibles sont-elles encore satisfaisantes? Fort probablement que non. Pourtant, étant donné que le traducteur fait face à des textes à faible redondance, à des choix de vocabulaire capricieux, à un style dense et allusif, à un langage figuré, ainsi qu'à des jeux de mots, des plaisanteries, des métaphores, de l'ironie et des contradictions qu'introduit l'inconscient dans le discours, il faut s'attendre à ce qu'il y

${ }^{14}$ Il faut surtout éviter de trop simplifier les choses. Ladmiral explique que Lacan a parfois aidé à faire implanter un néologisme : * Le concept freudien de Trieb (avec l'adjectif triebhaft, ou les composés en Trieb...) devrait, dans le cadre d'une terminologie cohérente et rigoureuse, être traduit par le néologisme français pulsion (avec l'adjectif pulsionnel), comme on a tendance à le faire maintenant, notamment depuis les énergiques remarques de J. Lacan • (Ladmiral, 1979, pp. 253-254).

Is « [D]ans un texte à faible redondance (c'est-à-dire, là où un nombre relativement petit de signes communiquent une grande quantité d'information) [...], le lecteur aura à sa disposition un plus grand éventail d'interprétation, donc de choix de traduction, que dans un texte hautement redondant, c'est-à-dire manifestement explicite, où les unités linguistiques sont très prévisibles " (Larose, 1987, p. 245).

${ }^{16}$ Ladmiral s'explique : * La diversité des registres où la psychanalyse a puisé son vocabulaire est fort grande : théorie de l'énergie, biologie, neurologie, psychologie * classique *, mythologie ou même langage des institutions... [...] Le discours scientifique de la psychanalyse a ceci de commun avec le discours poétique qu'il convient d'y intégrer la connotation à la dénotation quand on entreprend de le traduire * (Ladmiral, 1979, p. 251). 
ait des lectures divergentes, et la réécriture de la pensée de Lacan devient ainsi quasiment inévitable.

\section{(Ré)écriture de Lacan}

Patrick Mahony explore la question de la textualisation dans son essai $O n$ Translating Freud (1994). Il décrit les problèmes de traduction qui peuvent résulter de la subjectivité ou de l'appropriation personnelle de la langue commune - vision du monde individualisée et modelée par la langue maternelle - et de l'idéologie ${ }^{13}$, et il présente l'exemple d'un traducteur qui rend la psychanalyse viennoise de Freud plus " scientifique ", afin d'en faire un texte plus acceptable et donc compréhensible pour le lecteur anglophone (un peu comme Lacan a reformulé Freud afin de rendre plus conforme sa pensée à l'esprit français ${ }^{18}$ ). La subjectivité du traducteur risque donc de s'ajouter à la subjectivité du discours de l'auteur. L'altérité est ainsi subsumée sous la vision du monde sociale et personnelle du traducteur.

Mais, comme nous venons de voir, dès le départ, le texte de Lacan se prête mal à une * excellente " traduction (Ladmiral, 1979, p. 92). Pour sa part, Larose (1987, p. 245) affirme : " dès que le sens d'un texte est impossible (ou difficile) à cerner et dès qu'il y a interprétation [...], le texte perd son caractère objectivable puisque chaque traducteur réinvente

17 Ladmiral présente la problématique en d'autres mots, parlant plutôt d'« un affrontement théorique entre les "germanistes" et les "freudologues". Il y a là deux "camps" incarnant deux options traductologiques fondamentales : les uns privilégiant leur sensibilité au tissu même de la langue écrite par ce très grand styliste de l'allemand qu'était Freud, les autres se montrant essentiellement soucieux de faire apparaître la logique d'une construction théorique, et terminologique, à laquelle travaille l'œuvre du même Freud * (Kassaï et Ladmiral, 1988 , p. 4).

Is L'ironie : la réception initiale en France était semblable à la réception anglosaxonne. Selon Charbonneau. "L'important est que Lacan ait à soutenir [sa thèse] devant des médecins qui, lorsqu'ils sont favorables à la psychanalyse, voient la nécessité de la mater, de l'annexer, de la soutnettre à la science médicale *. Et plus loin, * Lacan accorde, on l'a vu, de ia valeur à la technique psychanalytique; en outre, il s'entend avec [les milieux médicaux] pour rejeter le symbolisme freudien " assez complexe et lointain " (Charbonneau, 1997, pp. 74-75). 
l'cuuvre selon sa propre lecture ». C'est précisément ici que l'inconscient peut s'insinuer. À ce sujet Mahony écrit (1994, p. 322) : * The siren call of the inexhaustible unconscious lures every psycho-analyst, monolingual or not, into being both translator and traitor. "On a vu que même Lacan en tant que traducteur avait \& trahi » Freud par moments (par exemple, ses multiples traductions de "Wo Es war, soll /ch werden "), si on n'accepte pas son argumentation qui veut qu'il n'y ait pas d'accord entre le verbe et le « moi qui parle ${ }^{\dagger 9}$, étant donné que la forme des verbes " soll, vienne et come " est la même à la première et à la troisième personnes. Dans sa réponse à Mahony, Sixel (1994, p. 326) ajoute que l'on pourrait dire la même chose au sujet du traducteur * who is always embedded in his own subjectivity ". Pour sa part, Ladmiral aborde la question de la psychanalyse de la traduction et du danger du refoulement dans l'acte traductionnel (Kassal et Ladmiral, 1988, p. 4). Malheureusement, l'étude de cette question dépasse les limites de notre travail. Toutefois, selon le psychanalyste, l'étiologie de la trahison en traduction se trouve plutôt au niveau de l'inconscient (surmoi) que dans les visions du monde et les représentations linguistiques différentes qui créent également une certaine subjectivité. Ceci peut avoir alors pour résultat une lecture ou une traduction différentes.

Regardons maintenant de plus près diverses façons d'aborder la traduction du Rapport du Congrès de Rome (1953) connu sous le titre de « Fonction et champ de la parole et du langage en psychanalyse " qui fait partie des Écrits de Jacques Lacan. D'une part, Anthony Wilden (1968, rév. 1981) s'est servi d'une approche traductionnelle qu'on pourrait

19 Wilden écrit une note de traducteur $\left(\mathrm{n}^{\circ} 110\right)$ à ce sujet : This linguistic phenomenon is mentioned again by Lacan in dealing with the Ich of Freud's "Wo es war solf Ich werden", deformed by the French translators into "Le moi doit déloger le ca "and originally rendered in English by "Where the id was there the ego shall be"... The reader should refer to "La Chose freudienne "(1956), pp. 237-238, for the development of Lacan's franslation" and its justification as: "La où c'était, peut-on dire, là où s'était, voudrions-nous faire qu'on entendît, c'est mon devoir que je vienne à être. "(Lacan, 1966, pp. 417-418) The created verb s'être being used to express "absolute subjectivity insofar as Freud properly discovered it in its radical ex-centricity" ... For Lacan it would seem that since "soll," "vienne" and "come" can each be read as either first or third person verb forms, they are not necessarily in agreement with the moi qui parle " (Wilden, 1981, p. 33). Voir également Benvenuto et Kennedy, 1986, p. 170. 
qualifier de métatexte. Il a placé en tête de sa traduction une introduction du traducteur (pp. vii-xix), où il a exposé les problèmes de traduction avec perspicacité. Cette introduction familiarise le lecteur avec l'arrière-plan gallique (Sartre, Wallon) de la pensée de Lacan et avec les influences germaniques (Freud, Husserl, Heidegger, Hegel) qui se sont exercées sur celle-ci. Comme Ladmiral l'a précisé, "Freud reste la référence primordiale, le seul Auteur (auctor) de la psychanalyse, celui qui fait autorité " (Ladmiral, 1979, p. 251). Wilden a fait suivre sa traduction (pp. 1-88) d'une abondance de notes (pp. 89-156) et d'un essai sur le discours de l'Autre (pp. 157-313). Le lecteur de cet ouvrage ne peut s'empêcher de se demander s'il ne s'agit pas plutôt d'une exégèse.

D'autre part, la traduction faite par Alan Sheridan de ce texte fait partie du volume Écrits : A Selection (1977). Sheridan a permis au lecteur américain de se familiariser avec la pensée de Lacan dans une traduction beaucoup plus accessible, grâce aux commentaires révélateurs et astucieux dans ses notes de traducteur, et sa traduction se lit plutôt comme une ceuvre originale. Ses traductions de Lacan jouissent d'une grande popularité auprès des étudiants de lettres anglaises, car on les considère accessibles.

Puisque Benvenuto et Kennedy, de même que Muller et Richardson, présentent et commentent la pensée de Lacan, ils ne proposent de nouvelles traductions que lorsqu'ils ne sont pas en accord avec les traductions existantes, ou lorsque aucune traduction n'existe. Ils comptent généralement sur les traductions de Wilden et de Sheridan.

L'observation la plus digne d'attention est qu'il y a désaccord entre les traducteurs sur la lecture de bon nombre de passages et de termes spécifiques (déjà au premier paragraphe, on trouve action-Wilden contre act-Sheridan (choix qui créera également un problème lorsqu'il s'agit de traduire Juranville!), awe-Wilden contre fright-Sheridan et lineamentsWilden contre face-Sheridan). À titre d'exemple qui saute aux yeux, Wilden a traduit a Fonction et champ de la parole et du langage en psychanalyse "par The Function of Language in Psychoanalysis, et Sarup par "The function of language in psychoanalysis", [o]ften called the "Rome report" (Sarup, 1992, p. xii). La première traduction de Sarup, proposée d'abord par Wilden, présente une simplification (qui risque d'induire en erreur) de la pensée de Lacan, ce demier faisant une distinction nette entre discours et langage. Sa deuxième traduction, surtout 
le choix de "report" est peu usuelle et risque de confondre le lecteur. Pour sa part, Sheridan l'a traduit de façon plus littérale comme The Function and Field of Speech and Language in Psychoanalysis. Benvenuto et Kennedy se réfèrent tout simplement au Rome Discourse, la référence la plus populaire même en français (le Discours de Rome de 1953). Lorsque ce titre se trouve cité par Assoun ou Juranville, par exemple, le traducteur doit donc choisir entre une traduction existante ou une nouvelle qu'il proposera. Toujours est-il qu'il commence souvent par se poser les questions suivantes : quelle traduction existante reproduit le plus précisément la pensée de Lacan? Quel titre le lecteur de Lacan reconnaîtra-t-il en anglais? $\mathrm{Y}$ a-t-il lieu d'en proposer une nouvelle?

Étant donné le vaste champ du savoir, le peu de redondance et l'insertion de l'inconscient dans la formulation des propos, ainsi que des cas d'ambiguîté et d'intraduisibilité, des lectures différentes ne doivent pas étonner. Dans cette prochaine section, nous nous référerons aux traductions de Wilden et de Sheridan déjà identifiées, et à la critique des traductions que font Benvenuto et Kennedy, ainsi que Muller et Richardson, dans leurs études qui portent sur la pensée de Lacan.

Dans sa traduction de The Four Fundamental Concepts of Psychoanalysis, Sheridan explique les cas d'ambiguitté lexicale dus à la polysémie en se servant des notes infrapaginales. Dans le passage suivant, le mot " souffrance " n'est pas traduit, car Sheridan n'a pas trouvé un seul mot anglais qui rende tous les sens français :

To this requirement correspond those radical points in the real that I call encounters, and which enable us to conceive reality as unterlegt, untertragen, which, with the superb ambiguity of the French language, appear to be translated by the same word-souffrance. Reality is in abeyance here. (Sheridan, 1977, pp. 55-56)

La note de Sheridan justifie sa décision de laisser "souffrance " en français : "In French, the phrase "en souffrance" means "in suspense", "in abeyance", "awaiting attention", "pending". It is this sense that translates the German word. "Souffrance" also means "pain", of course. Hence the ambiguity referred to by Lacan "(Sheridan, 1977, p. 56). Sheridan ne se croit en mesure de garder l'ambiguîté lexicale qui résulte de la polysémie du français qu'en retenant le vocable français et en le définissant. Lacan se sert de l'allemand dans ce passage, puisque comme 
on l'a déjà noté, il veut expliquer Freud, et il se réfère souvent à l'œuvre du " maitre " de langue allemande afin de communiquer l'esprit germanique. Toutefois, il semble que Lacan ait trouvé une bonne solution de traduction. En revanche, dans cet extrait on peut constater que Sheridan n'est pas toujours fidèle à la pensée de Lacan, car le traducteur a écrit " the superb ambiguity of the French language "alors qu'en français Lacan a écrit "ce qui en français se traduirait par le mot même, en sa superbe ambigułté dans la langue française, de souffrance ". Dans ce passage, Lacan se réfêre très précisément aux mots allemands, qui ont un champ sémantique non identique au champ sémantique du terme français (" unterlegt ", * untertragen " étant spécifique et * souffrance ", générique et polysémique) et ne fait pas référence à l'ambiguïté de la langue française en général (Lacan, 1973, p. 55). Nous avons donc également dans cet extrait un exemple de réécriture de Lacan par un traducteur, réécriture qui pourrait engendrer une discussion théorique sur l'ambiguité de la langue française et sur l'aptitude de celle-ci à rendre le discours de la psychanalyse.

Benvenuto et Kennedy (1986) participent à " l'enjeu de controverse dans la discussion ", icj " philosopho-analytique " (Ladmiral, 1979 , p. 92), en accusant Sheridan d'avoir mal traduit le titre * L'instance de la lettre " par * The Agency of the Letter ». Mais voici d'abord la justification proposée par Sheridan :

Lacan's use of the term * instance "goes well beyond Freud's * Instanz . It represents, one might say, an exploitation of the linguistic possibilities of the French equivalent of Freud's German term. In the absence of any exact equivalent of Lacan's French term, one is thrown back to the term used by Frewd's English translators, "agency ب. In Freud, the reference is most often to the three "agencies * of the id, ego and superego. In Lacan, one must bear in mind the idea of an " acting upon "even *insistence , as in the title of the essay, \& L'instance de la lettre *. (Sheridan, 1981, pp. 277-278)

Benvenuto et Kennedy (1986) traduisent par * The Instance of the Letter "et s'expliquent à ce sujet à la page 107 :

The English translator of Lacan has in our opinion incorrectly translated * instance * by * agency *. It would be more accurate to restore the old English word a instance "which is directly equivalent 
to the French, (and also to the German of Freud, Instanz). Instance in English means a pressing solicitation, an insistent request, urgency in speech or action, an urgent entreaty; as well as a trial, and the power or jurisdiction of a court, or the court itself. an authority which hos the power of decision.

après avoir analysé la notion d'agency à la page 47 :

In Freud's theory of the mind, the psychical apparatus was differentiated into a number of systems or wagencies", each of which had distinct properties and functions, but which interacted dynamically and in conflict with each other. ${ }^{*}$ Two theories of these agencies can be identified in Freud's writings.

*In Chapter 6 (p. 107) we argue that the old English word "instance " is more faithful to Freud's use of the German a Instanz " than *agency".

Cependant, Muller et Richardson ne touchent pas au titre. D'ailleurs, ils justifient leur décision dans une note qui suit le texte :

The translation of l'instance as "agency "suggests the active nature of the letter in the unconscious, but not the quality of this action. In the first English translation of this essay, Miel (1966) uses ainsistence ", conveying the autonomous quality of this agency. (Miel's translation is one we have drawn on, as Sheridan apparently also has in his transiation of Écrits: A Selection). In French, l'instance means * entreaty * "solicitation * * urgency * " earnestness * and "instance ", with the last strengthened by the notion of the "in-standing * or persistence of the letter. (Muller et Richardson 1982, p. 182)

Curieusement, dans ce passage Muller et Richardson présentent des arguments par inadvertance pour " instance ". Mais la polémique autour de la traduction d' $\alpha$ instance " ne se limite pas à ce titre. Lacan se réfère dans ses divers écrits à " instance subjectale, instance discursive, instance d'altérité, instance du "désir", instance symbolique ». Et lorsque le traducteur d'Assoun rencontre "l'instance discursive de l'Autre ", par exemple (Assoun, 1992, p. 124), il se demande quelle traduction parmi les trois proposées (agency, instance, insistence) - parmi peut-être d'autres - est celle qui reproduit le mieux la pensée de Lacan et que le lecteur s'attend à lire dans la traduction anglaise.

Ce qui suit est encore un exemple d'un passage que Sheridan considère intraduisible et qu'il supprime dans sa traduction - ainsi 
réécrivant Lacan -, quoiqu'il l'inclut à titre de note infrapaginale :

There follows a passage in which Lacan comments on the use in French of the pleonastic ne ", that is, the " ne "used without the usually accompanying " pas " "que * or " jamais ", etc. Since the passage includes examples of this use in French, it is strictly untranslatable. I therefore give it below in the original:

Je suis, que je sache, avant que je ne me réveille - ce ne dit explétif, déjả dans tel de mes écrits désigné, est le mode même de présence de ce je suis d'avant le réveil. Il n'est point explétif, il est plutôt l'expression de mon impléance, chaque fois qu'elle a à se manifester. La langue, Ja langue française le définit bien dans l'acte de son emploi. Aurez+vous fini avant qu'il ne vienne? - cela m'importe que vous ayez fini, à Dieu ne plaise qu'il vint avant. Passerezz-vous, avant qu'il vienne - car, déjà, quand il viendra, vous ne serez plus là. (Sheridan, 1977, pp. 5657)

Examinons maintenant la difficulté de traduction qui se présente lorsque les traductions existantes ne semblent pas s'accorder sur le sens d'une formule de Lacan. Dans son article * Le sujet et l'Autre chez Levinas et Lacan n, Paul-Laurent Assoun (1993, p. 126) cite Lacan (Écrits, 1966, p. 818) : « signifiant d'un manque dans l'Autre, inhérent à sa fonction même d'être le trésor du signifiant ». Le traducteur d'Assoun qui consulte la traduction de Sheridan (Écrits, 1977, p. 316) trouve la traduction suivante : a signifier of a lack in the Other, inherent in its very function as the treasure of the signifier ". Par contre, il trouvera que Sarup (1992, p.112), entre autres, propose de rendre " trésor " par " treasure house " ou bien par * treasury " sans indiquer la source. Par la suite, le traducteur se réfère à Benvenuto et Kennedy $(1986$, p. 176) qui discutent du sens du "trésor du signifiant ", mais ne critiquent pas la traduction proposée par Sheridan (on a déjà vu qu'ils n'hésitent pas à le faire ailleurs) et même ajoutent : "Lack is to be seen as inherent in the very function of the Other as the "treasure" of the signifier * (p. 176). La traduction apparente de " trésor " est " treasure "; par contre, "treasury" ou * treasure house " présente une lecture beaucoup moins littérale du texte de départ et révèle une compréhension divergente du texte de Lacan. Ailleurs, Sheridan traduit l'" angoisse " de Lacan par " anxiety ", tandis que Benvenuto et Kennedy critiquent sa traduction et proposent " anguish " (sens kierkegaardien). De tels exemples isolés peuvent déjà susciter une discussion théorique sur le sens profond de la pensée de Lacan. 
Quelle solution : de multiples traductions ou une " traduction canonique $n$ ?

Traduire * Le sujet et l'Autre chez Levinas et Lacan "d'Assoun et "L'Éthique avec la psychanalyse * de Juranville présente des difficultés de traduction liées aux références textuelles à la pensée de Lacan. Parfois les difficultés semblent être d'une grande banalité. Par exemple, Assoun se sert de la majuscule dans son titre (Autre), ce qui se fait plutôt rarement en français. Mais étant donné qu'il s'agit du grand Autre qui s'écrit avec une majuscule, alors que le petit autre s'écrit avec une minuscule, Assoun ne fait que respecter la terminologie (et l'orthographe) dont se sert Lacan afin de se faire comprendre. Le contraire est la norme en anglais. $D$ 'habitude, les mots importants des titres (noms, verbes) commencent par la majuscule. Mais dans ce cas-ci, afin de distinguer le grand Autre du sujet, il a fallu écrire subject avec une minuscule et garder Other avec une majuscule. Le résultat "The subject and the Other in Levinas and Lacan * apparaît de prime abord comme une erreur typographique. Mais cette apparente idiosyncrasie typographique a une charge sémantique très lourde et influence à partir du titre la lecture de l'essai d'Assoun, car le titre anglais fait savoir qu'il s'agit de la relation entre le sujet et le grand Autre.

En fin de compte, c'est la moindre des difficultés de la traduction de ces textes, puisque généralement les difficultés de traduction s'aggravent en fonction de la multiplication de lectures ou d'interprétations, comme les solutions différentes proposées par Wilden et Sheridan en témoignent (pensons à act/action, awe/fright, treasure/treasury/treasury house, anxiety/anguish). Et l'exemple (langue de départ) évoqué plus haut ne présente pas la possibilité de multiples lectures (par contre, si le traducteur avait décidé d'écrire Subject et Other avec des majuscules, le contraire aurait été vrai dans la langue d'arrivée!). Étant donné que les lecteurs de ces textes seront en grande partie également les lecteurs de Lacan, il se peut que ces lecteurs français et américains veuillent y reconnaître un terme, un titre ou une expression lacaniens et cherchent une terminologie et un style normalisés tant en anglais qu'en français.

Néanmoins la question reste sans réponse : les écrits de Lacan étant difficiles, le traducteur de ces textes doit-il tout simplement écarter les traductions existantes et proposer la sienne qui peut - mais ne doit 
pas - se conformer à une traduction existante qui fasse autorité? Il ne s'agissait pas ici de formuler une argumentation contre la traduction de Lacan, mais plutôt d'explorer les solutions éventuelles à notre dilemme traductionnel. Notre réponse : la difficulté des écrits de Lacan justifie que plusieurs traductions soient proposées et que, comme Ladmiral l'écrit $(1979$, p. 92$)$, * celles-ci deviennent un enjeu de controverses dans la discussion philosophique ». Déjà Assoun et Juranville participent à la réécriture de Lacan, et donc à cette même discussion que la traduction déclenche. Toujours est-il que les écrits de Lacan sont difficiles à pénétrer. Il se peut, par ailleurs, qu'il y ait résistance socio-culturelle, linguistique ou inconsciente à sa pensée ou à la forme de sa pensée, ce qui risque également d'entraîner différentes interprétations et différentes traductions, à l'image des discussions théoriques suscitées par sa pensée. Dans ce cas-ci, on écartera donc la primauté d'une traduction canonique et on considérera plutôt la multiplication des traductions comme un phénomène qui enrichit la compréhension de la pensée de Lacan. ${ }^{20}$

Université de Moncton

\section{Références}

ASSOUN, Paul-Laurent (1993). " Le sujet et l'Autre chez Levinas et Lacan ». Rue Descartes 7, pp. 123-145.

BENVENUTO, Bice et Roger KENNEDY (1986). The Works of Jacques Lacan. New York, St. Martin's Press Inc.

CHARBONNEAU, Marie-Andrée (1997). Science et métaphore : enquête philosophique sur la pensée du premier Lacan (1926-1953). Québec, Presses de l'Université Laval.

\footnotetext{
2u'Je tiens à remercier Ginette Michaud et Jean-Jacques Hamm d'avoir lu et commenté le présent article, Marilyn Gaddis Rose, Rosemary Arrojo, Louis Jolicoeur et Susan Ingram pour une discussion très fructueuse à la suite de la lecture de sa version embryonnaire lors du Congrès annuel de l'ACT du 3 juin 1997 à Saint-Jean, Terre-Neuve. De plus, je souhaite remercier Sarah Harasym pour son invitation à participer en tant que traductrice à ta publication sous sa direction, Levinas and Lacan : The Missed Encounter (Albany, State University of New York Press, 1998) - expérience qui a inspiré le présent article. Enfin, merci à Matthieu LeBlanc d'avoir fait une lecture d'épreuve.
} 
DUFRESNE, Todd (dir.) (1997). Returns of the 'French Freud' : Freud, Lacan, and Beyond. New York/Londres, Routledge.

HOMMEL, Susanne (1988). "Wo Es war, soll lch werden : interprétations lacaniennes $\%$. Dans Traduction et psychanalyse (Kassaï et Ladmiral dir.). Paris, Le Coq-Héron et Contrastes, A-7, $n^{\circ}$ 105, pp. 6-9.

HONDERICH, Ted (dir.) (1995). The Oxford Companion to Philosophy. Londres, Oxford University Press.

JURANVILLE, Alain (1986). " L'éthique avec la psychanalyse *. Corps écrit, 19, pp. 97-111.

KASSAI, Georges et Jean-René LADMIRAL (dir.) (1988). Traduction et psychanalyse. Paris, Le Coq-Héron et Contrastes, A-7, $n^{\circ} 105$.

LACAN, Jacques (1981). The Four Fundamental Concepts of PsychoAnalysis (tr. Alan Sheridan). New York/Londres, W.W. Norton \& Co.

- (1977). Écrits : A Selection (tr. Alan Sheridan). New York/Londres, W.W. Norton \& Co.

- (1973). Séminaire $I X$ de 1964 : Les quatre concepts fondamentazx de la psychanalyse (Jacques-Alain Miller dir.). Paris, Éditions du Seuil.

- (1968). The Language of the Self: The Function of Language in Psychoanalysis (tr. Anthony Wilden). Baltimore, The Johns Hopkins Press. (Révisé en 1981 sous le titre de Speech and Language in Psychoanalysis).

- (1966). "Fonction et champ de la parole et du langage en psychanalyse n. Écrits. Paris, Seuil, pp, 237-322.

- (1966), « La chose freudienne *. Ecrits. Paris, Seuil, p.401-436.

LADMIRAL, Jean-René (1979). Traduire : Théorèmes pour la traduction, Paris, Payot, « Petite Bibliothèque Payot n.

- (1979). * Note sur le discours psychanalytique *. Appendice I de 
Traduire : théorèmes pour la traduction, Paris, Payot, " Petite Bibliothèque Payot ", pp. 249-257. (Cette N. d. T. a suivi la première édition de sa traduction du livre de Erich Fromm, La Crise de la psychanalyse (1971), pp. 283-290).

LAROSE, Robert (1987). Théories contemporaines de la traduction. Québec, Presses de l'Université du Québec.

LEFEVERE, André (1992). Translation, Rewriting and the Manipulation of Literary Fame. Londres/New York, Routledge.

MAHONY, Patrick (1994). * Hermeneutics and ldeology : On Translating Freud w, Meta, 39/2, 316-324.

MULLER, John P. et William J. RICHARDSON (1982). Lacan and Language : A Reader's Guide to Écrits. New York, International Universities Press Inc.

SARUP, Madan (1992). Jacques Lacan. Toronto, University of Toronto Press, * Modern Cultural Theorists *.

SIMON, Sherry (1996). * Missed Connections : Transporting French Feminism to Anglo-America *. Gender in Translation, Londres/New York, Routledge, pp. 86-110.

SIXEL, Margrit M. (1994). " Restoring Freud: Some Notes on P. Mahony's Essay ", Meta, 39/2, pp. 325-327.

TESTA, Bart (1997). " Jacques Lacan : Harbinger of Posthumanism *. The Globe \& Mail, 23 aout, p. D9.

TURKLE, Sherry (1981). Psychoanalytic Politics : Freud's French Revolution. Cambridge (Massachusetts), The MIT Press.

RÉSUMÉ : (Ré)écriture du discours psychanalytique lacanien en traduction-Cette étude se veut une réflexion sur l'apport de la réécriture des textes psychanalytiques de Jacques Lacan à la réception de sa pensée. Jean-René Ladmiral est d'avis que les textes psychanalytiques " difficiles ", de même que les textes philosophiques a profonds " et " obscurs ", se prêtent mal une seule interprétation, invitant ainsi 
" plusieurs traductions \%. Ces traductions différentes découlent inévitablement des lectures multiples résultant d'une riche intertextualité qui s'inspire de plusieurs disciplines : la médecine, la psychiatrie, la psychologie, la psychanalyse, la philosophie. Les influences germaniques (Freud, Husserl, Hegel) ainsi que françaises (Saussure, Lévi-Strauss, Sartre) contribuent également à tisser l'intertexte lacanien, rendu davantage complexe par les manifestations de l'inconscient dans le discours. Au lieu de condamner la multiplication de lectures et de traductions, nous proposons que ces lectures et traductions différentes enrichissent et approfondissent notre compréhension de la pensée complexe de Lacan.

ABSTRACT : (Re)writing Lacan's Psychoanalytic Discourse in Translation-This study aims to come to terms with how rewriting Jacques Lacan's psychoanalytic texts contributes to understanding his thought. Jean-René Ladmiral believes that "difficult" psychoanalytic texts, like "profound" and "obscure" philosophical writings, invite multiple translations, since they do not lend themselves to a single reading. These different translations reflect the multiple readings resulting from the rich intertext that draws its inspiration from several disciplines: medicine, psychiatry, psychology, psychoanalysis, and philosophy. Germanic (Freud, Husserl, Hegel) and Gallic (Saussure, Lévi-Strauss, Sartre) influences have also been woven into the fabric of Lacan's intertext made yet more complex through the manifestation of the unconscious in his discourse. Rather than condemn the number and variety of readings and translations, we argue that they afford the opportunity to enrich and deepen our understanding of Lacan's complex thought

Denise Merkle : Département de traduction et des langues, case 30, Faculté des arts de l'Université de Moneton, Moncton (NouveauBrunswick) E1A 3E9

Courriel : merkled@umoncton.ca. 